\title{
A Pinhole Camera for Radiation Research in Dentistry
}

\author{
ALBERT G. RICHARDS \\ School of Dentistry, University of Michigan, Ann Arbor, Michigan
}

This paper describes the design and theory of a pinhole camera which produces simultaneously radiographs and photographs of any object which emits X-radiation and reflects visible light. Such a camera is very useful for radiation research in dentistry because both the patient and the $\mathrm{X}$-ray apparatus emit $\mathrm{X}$-radiation and reflect visible light while the patient is examined with X-rays.

\section{EXPERIMENTAL METHODS}

Design of pinhole camera.-The pinhole camera is essentially a box containing both a photographic film and a radiographic film (Fig. 1). The material and thickness of the walls of the camera are chosen to limit the exposure of the films to only those rays that can enter the camera through a very small opening in the wall opposite the films. The images will be inverted and reversed on the films, just like images produced by conventional cameras. Because X-rays and visible light both travel in straight lines from their points of origin, the pinhole camera is capable of recording the sources of both forms of radiation.

The walls of the camera must be both opaque and radiopaque,* to protect the films from undesirable exposure to light and to $\mathrm{X}$-rays generated with voltages as high as $90 \mathrm{KvP}$. These conditions are fulfilled adequately when the walls of the camera are made of lead, $\frac{3}{32}$ inch thick.

The pinhole camera, as shown diagrammatically in Figure 1, was designed so that the entire pinhole assembly could be removed and replaced with another assembly containing openings of other sizes.

The details of the pinhole opening through which the radiation must pass to enter the camera are shown in Figure 2. The pinhole assembly is formed by sandwiching a thin foil of aluminum between two disks of lead.

Tapered openings in the disks of lead form the radiographic pinhole opening through which X-rays can enter the camera. The proper amount of taper in the pinhole is determined by the ratio of the distance between the pinhole and the film to the diagonal dimension of the film. Aluminum foil is very radiolucent; $\uparrow$ hence the $\mathrm{X}$-rays pass through the foil that is in the opening of the disks of lead. The minute

This investigation was supported by U.S.P.H.S. research grant D-1116 from the National Institute of Dental Research, National Institutes of Health.

Received for publication April 6,1961.

* Radiopaque: objects and tissues that are relatively opaque to, or resist the passage of, $\mathrm{X}$-radiation are classified as radiopaque.

† Radiolucent: objects and tissues that are relatively translucent to, or permit the passage of, $\mathrm{X}$-radiation are classified as radiolucent. 


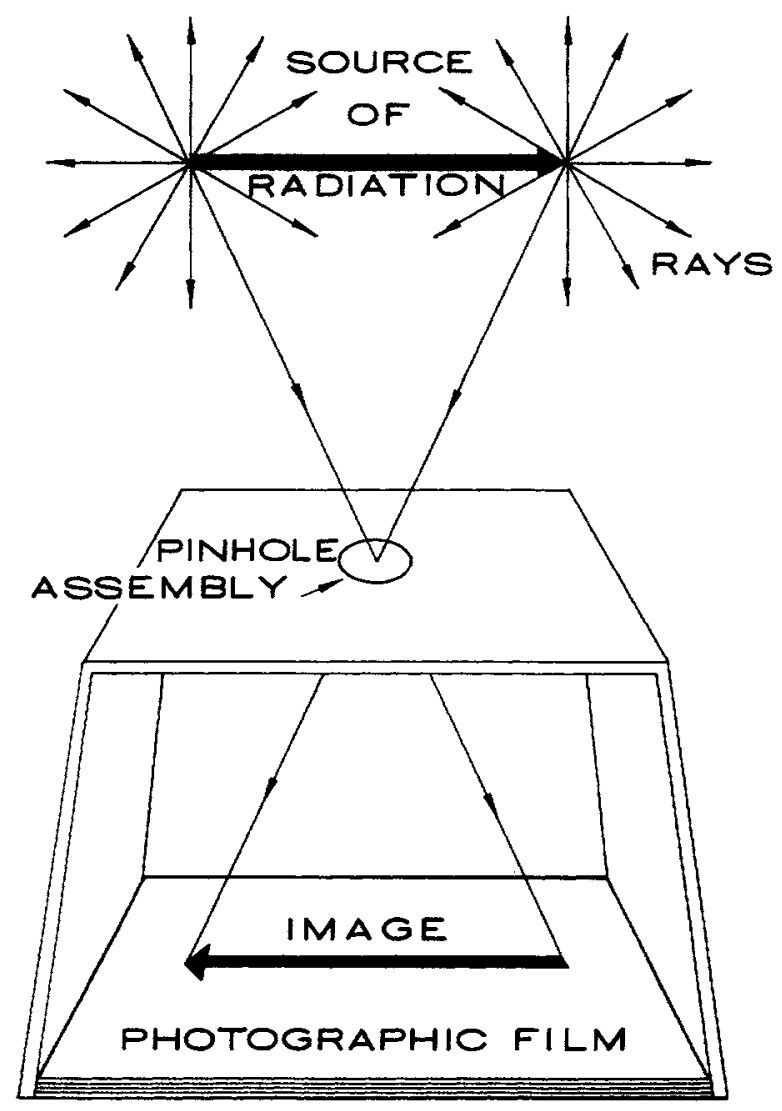

FIG. 1.-Pinhole camera with front wall removed. From all points on the source of radiation, rays are either emitted or reflected in every conceivable direction. Some of these rays enter the camera through the pinhole opening and record the image of the source on one of the two films contained within the camera. The walls of the camera can be made of lead.

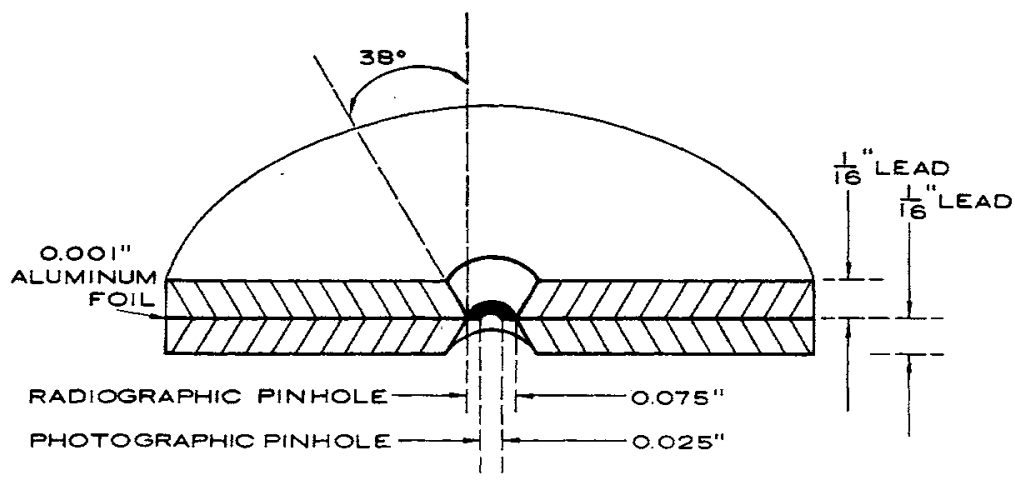

FIG. 2.-Pinhole assembly (vertical view). The pinhole camera contains two concentric pinhole openings. A minute opening in a thin sheet of aluminum foil allows rays of visible light to enter the camera, while somewhat larger openings in two sheets of lead serve to admit rays of X-radiation. 
opening in the foil of aluminum is concentric with the tapered opening of the disks of lead. Both lead and aluminum are opaque to visible light; therefore, the opening in the foil alone serves as the photographic pinhole.

An expanded view of the film assembly is presented in Figure 3. The film assembly is a combination of a cut-film holder and a cassette with intensifying screens. The rays of visible light, which enter the camera through the photographic pinhole, expose the photographic film. No visible light rays penetrate deeper than the magnesium sheet in the film assembly; therefore, the radiographic film is protected from this source of exposure. The radiographic film is exposed by the direct action of the Xradiation that reaches it and by the fluorescent light from the intensifying screens which are activated by the X-radiation. Portrait pan film* has been used successfully in the camera because of its great sensitivity to light and its low sensitivity to the $\mathrm{X}$-radiation that penetrates the various layers of the film assembly. The combination of intensifying screens $\dagger$ and $\mathrm{X}$-ray film $\ddagger$ has been used successfully in the camera to record radiographic images.

Satisfactory photographs have been made with the pinhole camera with exposures ranging between 35 to 60 minutes. Radiographic exposures made with radiation generated with $90 \mathrm{KvP}$ have ranged between 1,500 and 4,500 milliampere seconds. When the photographic exposure is completed before the radiographic exposure, the photo-

* Kodak. † † Patterson "Lightning Special." $\quad \ddagger$ Kodak "Royal Blue."

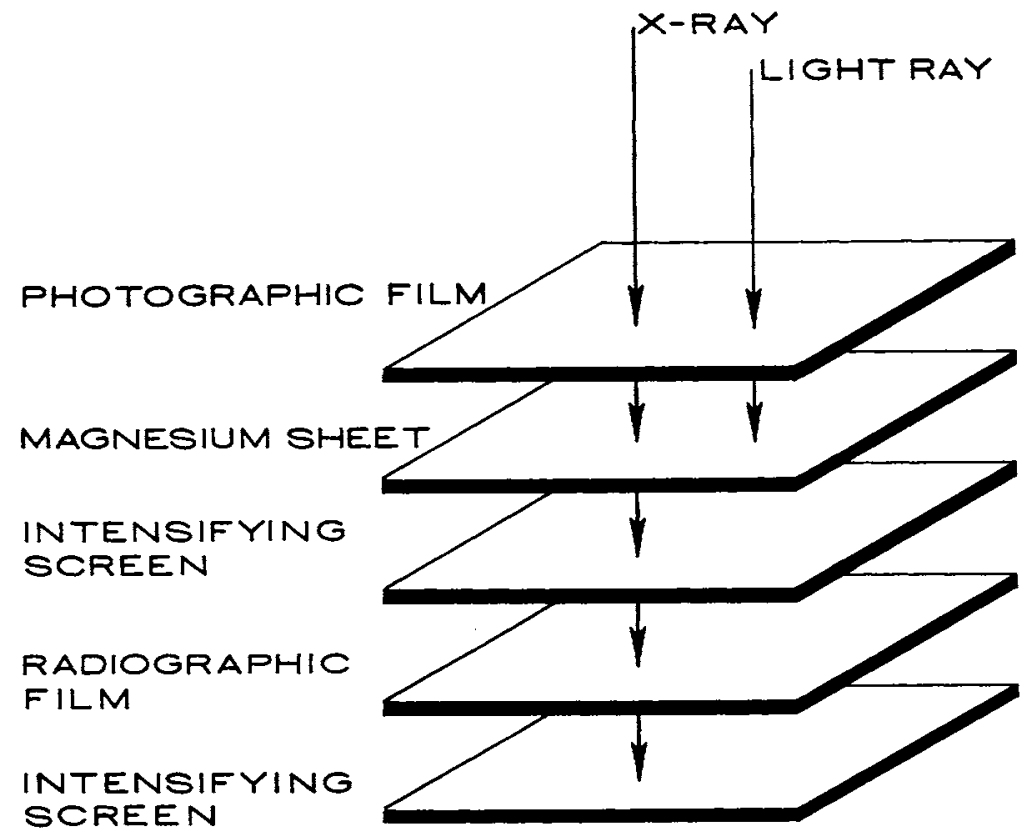

FIG. 3.-Film assembly (expanded view). The film assembly consists of a radiographic film located between two intensifying screens in a cassette. A photographic film is fastened onto the magnesium front surface of the cassette. The radiographic film is exposed in the cassette by the $\mathrm{X}$-rays that enter the camera, while the photographic film is exposed mainly by the visible light rays and to a far lesser extent by the $\mathrm{X}$-rays. 
graphic exposure is terminated simply by covering the pinhole assembly with a piece of thin, opaque plastic tape, and the radiographic exposure is allowed to continue until completion.

Dental X-ray machines are not designed for continuous operation at high voltage and current values, although some can be operated continuously with very low currents, provided that forced cooling is supplied to the head of the machine. Most equipment cannot be operated at high voltage and current values with more than a 6.5 per cent duty cycle, which means that, out of every minute, the apparatus can be operated no more than 6.5 per cent of the time, or 4 seconds. Non-operation of the apparatus during the remaining 56 seconds of each minute is necessary to allow for cooling of the anode. The duty cycle of an individual piece of equipment can be learned from the manufacturer of the equipment.

\section{THEORY OF THE PINHOLE CAMERA}

Time of exposure.-With any point source of radiation, the intensity of the radiation varies inversely as the square of the distance from the source. Therefore, the time of exposure for a film exposed in a pinhole camera to radiation from a point source is a function of the square of the distance from the source to the film and is independent of the diameter of the pinhole opening (Fig. 4).

Quite different conditions govern the time of exposure when the source of radiation is a large object that reflects or emits radiation of uniform intensity from every point. Under these conditions, the intensity of the radiation that exposes each point on the film is directly proportional to the square of the diameter of the pinhole opening and inversely proportional to the square of the distance from the pinhole to the film (Fig. 5 ). The time of exposure of the film is therefore a function of the square of the distance from the pinhole to the film divided by the square of the diameter of the pinhole opening and is independent of the distance from the source to the pinhole opening in the camera, provided that the source is large enough.

Enlargement.-With pinhole cameras, just as with conventional cameras, enlarged images, reduced images, or images of natural size can be recorded. Figure 6 indicates that the size of the image is directly proportional to the difference between the pinholefilm distance and the source-pinhole distance and inversely proportional to the sourcepinhole distance.

Resolution.--The theoretical limit on resolution of two point sources is directly proportional to the product of the pinhole diameter and the source-film distance and inversely proportional to the pinhole film distance (Fig. 7).

\section{RESULTS}

Figure $8, A$, is a photograph, obtained with the pinhole camera, of the relationship of the $\mathrm{X}$-ray cone and the phantom's head during the posterior bite-wing examination. Figure $8, B$, is a radiograph produced by radiation scattered from the cone and the phantom head. By viewing the superimposed photograph and radiograph (Fig. 8, $C$ ), the location of the invisible sources of $\mathrm{X}$-radiation portrayed in the radiograph can be oriented easily with respect to the visible details in the photograph.

Figure $9, A, B$, and $C$, portrays the photograph, radiograph, and the combination of the two pictures of the cone and head of an X-ray machine. Scattered X-radiation 


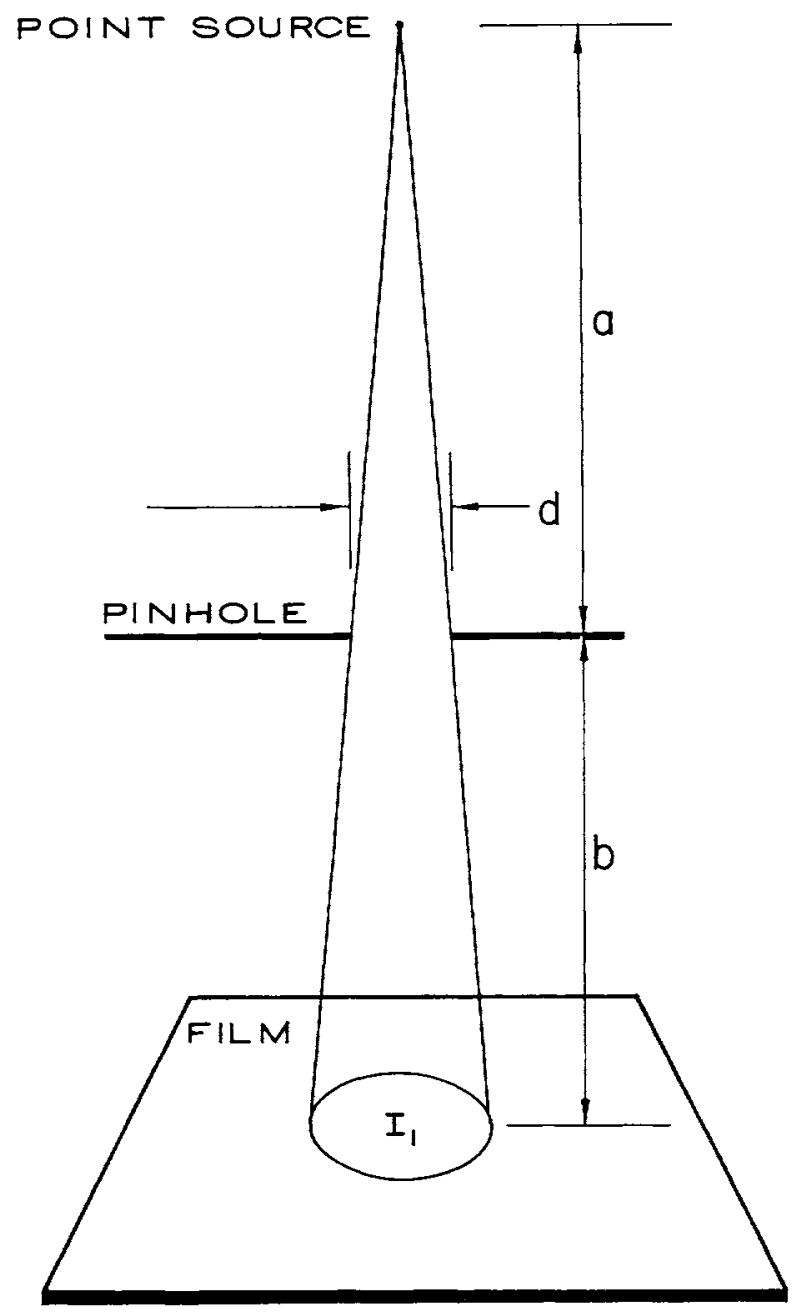

$a=$ Source to pinhole distance,

$b=$ Pinhole to film distance,

$d=$ Diameter of pinhole opening ,

$I_{1}=$ Intensity of radiation from point source as it exposes the film ,

$T_{1}=$ Time of exposure of film ,

$k_{1}=\mathrm{A}$ constant depending on the source,

$l_{1}=$ A constant depending on film sensitivity,

$f_{1}=$ A mathematical function ,

$I_{1}=\frac{k_{1}}{(a+b)^{2}}$,

$T_{1}=\frac{l_{1}}{I_{1}}=\frac{l_{1}}{k_{1} /(a+b)^{2}}=\frac{l_{1}(a+b)^{2}}{k_{1}}=f_{1}(a+b)^{2}$,

where

$\frac{l_{1}}{k_{1}}=f_{1}$ (a function of the intensity of source and film sensitivity) .

FIG. 4.-Time of exposure. Radiation from a point source, located at a distance $(a+b)$ from the film, reaches the film with intensity $I_{1}$. This intensity varies inversely with the square of the sourcefilm distance. The diameter $(d)$ of the pinhole and the distances $(a)$ and $(b)$ that the pinhole is located from the source and film, respectively, determine the size of the area of exposed film. The time of exposure of the film is some function of $(a+b)^{2}$ which takes into account the original intensity of the source and the sensitivity of the film. 
LARGE, UNIFORMLY INTENSE SOURCE OF RADIATION

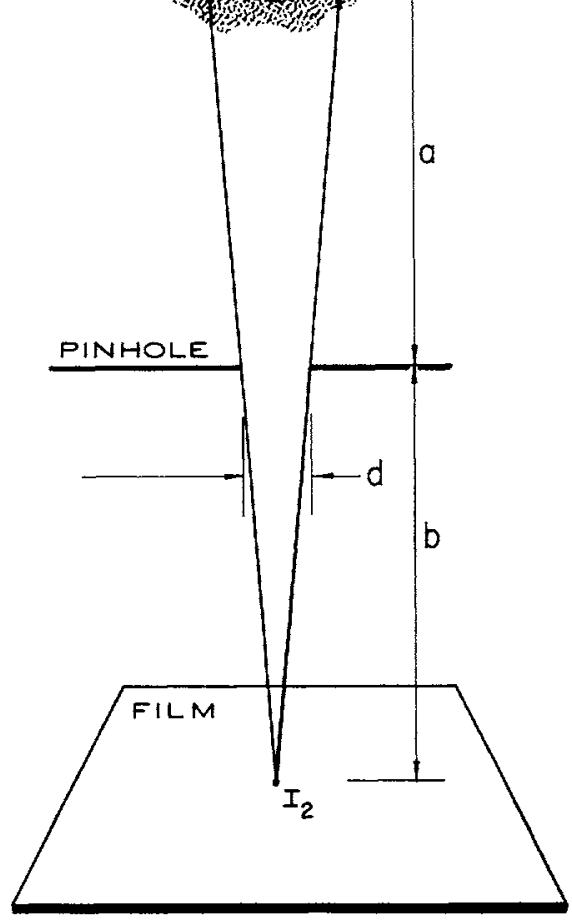

$I_{2}=$ Intensity at the film from all radiation from within the dotted circle on large source that can expose a single point on the film,

$T_{2}=$ Time of exposure of film,

$k_{2}=\mathrm{A}$ constant depending on the source,

$l_{2}=\mathrm{A}$ constant depending on film sensitivity,

$f_{2}=$ A mathematical function,

$I_{2}=k_{2} \frac{d^{2}}{b^{2}}$

$T_{2}=\frac{l^{2}}{I^{2}}=\frac{l_{2}}{k_{2}\left(d^{2} / b^{2}\right)}=\frac{l_{2} b^{2}}{k_{2} d^{2}}=f_{2} \frac{b^{2}}{d^{2}}$,

where $\quad \frac{l_{2}}{k_{2}}=f_{2}$ (a function of the intensity of source and film sensitivity).

FIG. 5.--Radiation from a large, uniformly intense source, located at a distance $(a+b)$ from the film, reaches a point on the film with intensity $I_{2}$. This intensity varies directly with the square of the pinhole's diameter $(d)$ and inversely with the square of the pinhole-film distance $(b)$. The time of exposure of the film is some function of $(b)^{2} /(d)^{2}$ which takes into account the original sensitivity of the film. 
apparently originated in the pointed plastic cone and in the filter and other structures located behind the lead diaphragm.

The profile view of the mandibular anterior region, which is presented as Figure 10, was produced in the pinhole camera by the scattered $\mathrm{X}$-radiation emanating from the cone and phantom head. The non-symmetrical image of the scatter from the cone indicates that the center of the X-ray beam did not coincide with the tip of the cone. The amount of scatter from the soft tissues of the patient is apparently greater than that from the osseous tissues.

Figure 11 is a radiograph, made with the pinhole camera, of the head of a phantom on which the maxillary central incisors region was examined with $\mathrm{X}$-radiation. Images

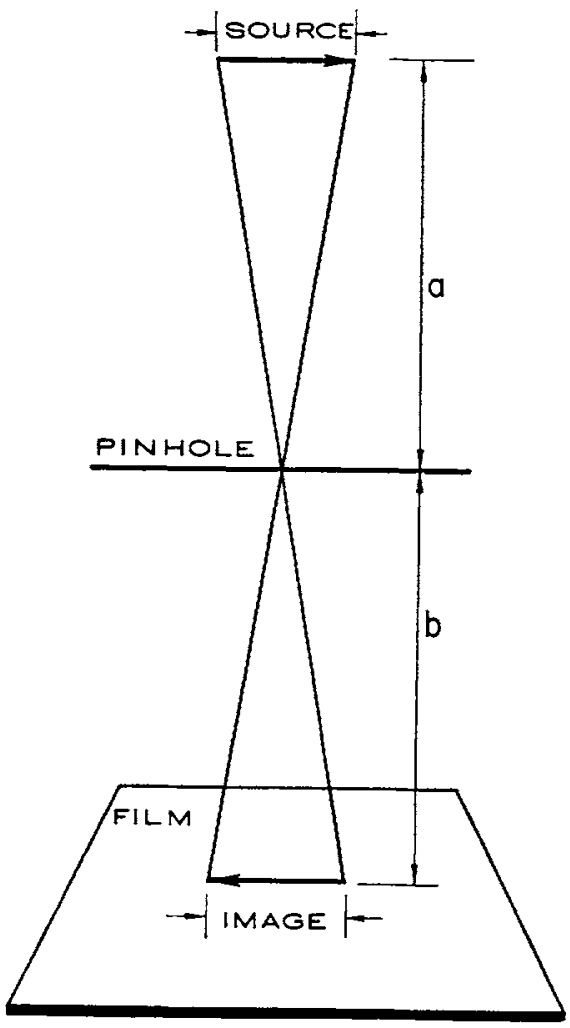

Per cent enlargement $=\frac{\text { Image size }- \text { Source size }}{\text { Source size }} \times 100=\frac{b-a}{a} \times 100$.

EXAMPLE: $\quad a=6^{\prime \prime}, b=4^{\prime \prime}$.

Per cent enlargement $=\frac{4-6}{6} \times 100=-33 \frac{1}{3}$ per cent, or the image is one-third smaller than the source.

FIG. 6.-Enlargement of image. The two constructed triangles, the apices of which meet at the pinhole, are similar triangles; therefore, the size of the image is to the size of the source as the pinhole-film distance $(b)$ is to the pinhole-source distance $(a)$. From these ratios, the per cent enlargement of the image is found to be proportional to one hundred times $(b-a) / a$. 


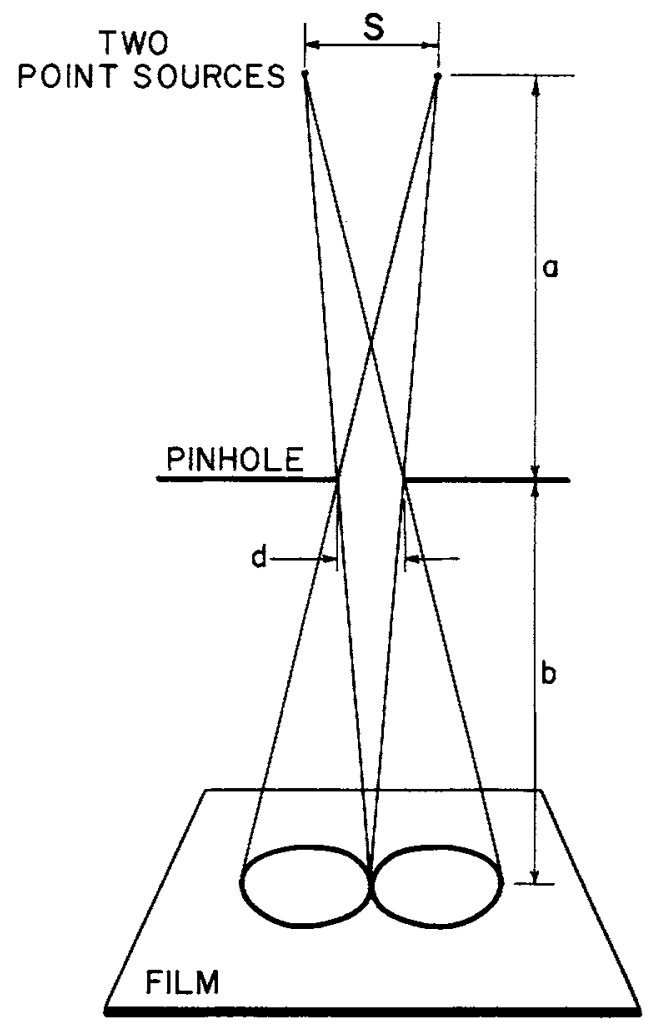

Resolution:

$S=$ Minimum distance by which two point sources can be separated and still produce separate images of the pinhole on the film,

$$
\begin{aligned}
\frac{S}{a+b} & =\frac{d}{b}, \\
S & =\left(\frac{a+b}{b}\right) d .
\end{aligned}
$$

EXAmple: $\quad a=6^{\prime \prime}, b=4^{\prime \prime}$.

For photography, $d=0.025^{\prime \prime}$,

For radiography, $d=0.075^{\prime \prime}$.

Photographic resolution:

$$
S_{p}=\left(\frac{6+4}{4}\right) 0.025=0.0625^{\prime \prime} .
$$

Radiographic resolution:

$$
S_{r}=\left(\frac{6+4}{4}\right) 0.075=0.1875^{\prime \prime}
$$

FIG. 7.-Resolution of two point sources. The resolution or resolving power of the pinhole camera is indicated by the minimum separation $(S)$ of two sources of radiation whose images will record distinct and separate on the film. This distance $(S)$ is proportional to the product of the diameter of the pinhole and the source-film distance, divided by the pinhole-film distance. 

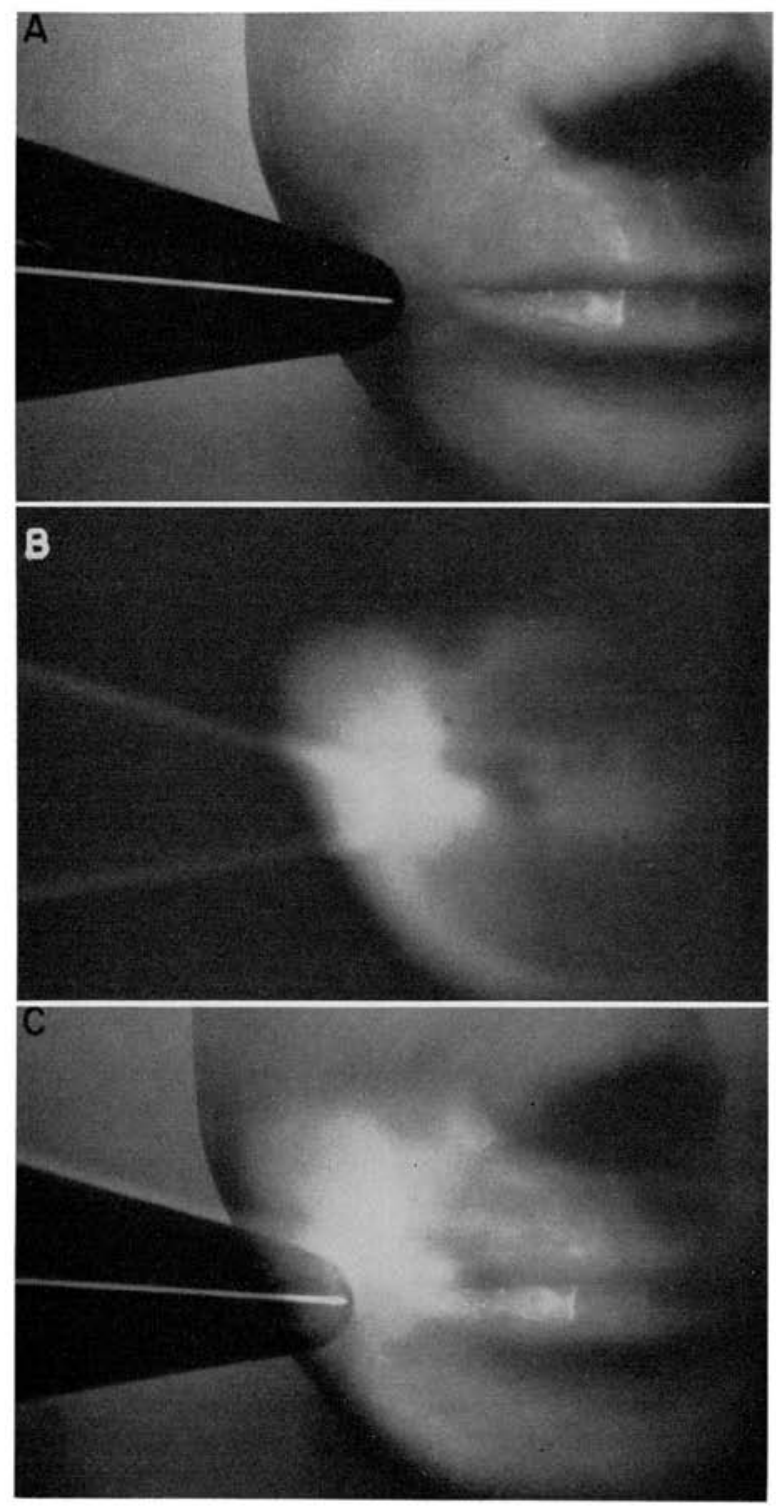

FIG. 8.-A phantom head was examined with the posterior bite-wing technique. This figure portrays the results obtained with a pinhole camera containing both photographic and radicgraphic films. $A$ is the photographic result, $B$ is the radiographic result, and $C$ is a combination of the photograph and radiograph. The $\mathrm{X}$-ray exposure used in producing this radiograph was approximately one hundred thousand times larger than would be given a living patient. 

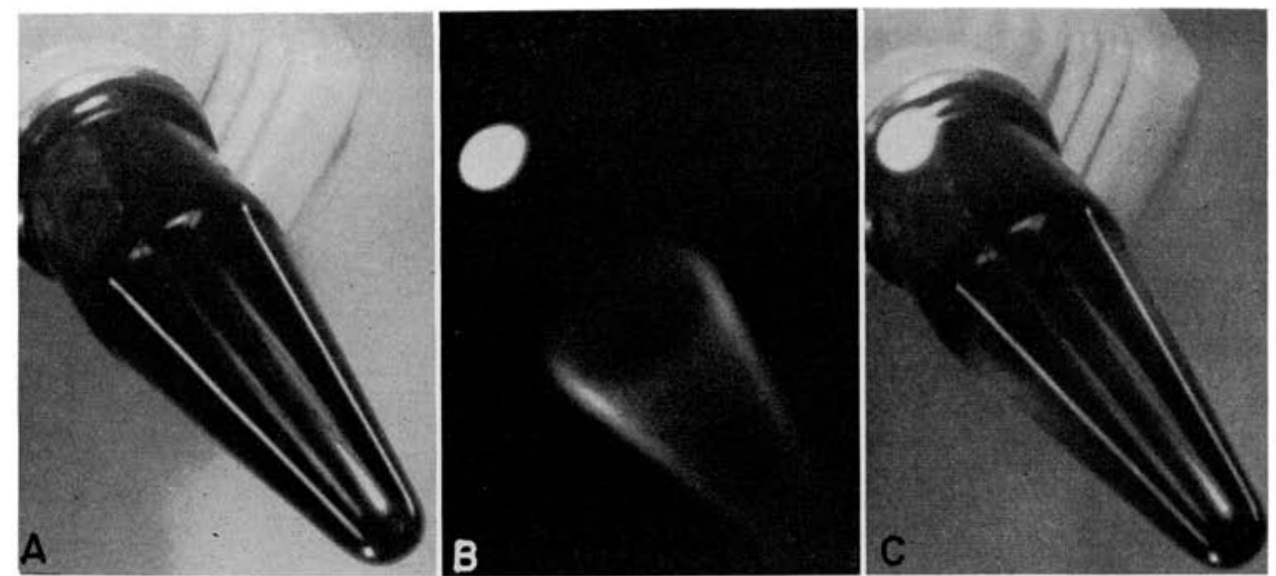

Fig. 9.-A pictorial investigation of the head of a dental X-ray apparatus was made with a pinhole camera containing both photographic and radiographic films. $A$ is the photographic image, and $B$ is the radiographic image recorded by the pinhole camera. Reflected light produced image $A$, while scattered $\mathrm{X}$-radiation produced image $B$. Image $C$ is the superimposed combination of photograph $A$ and radiograph $B$.

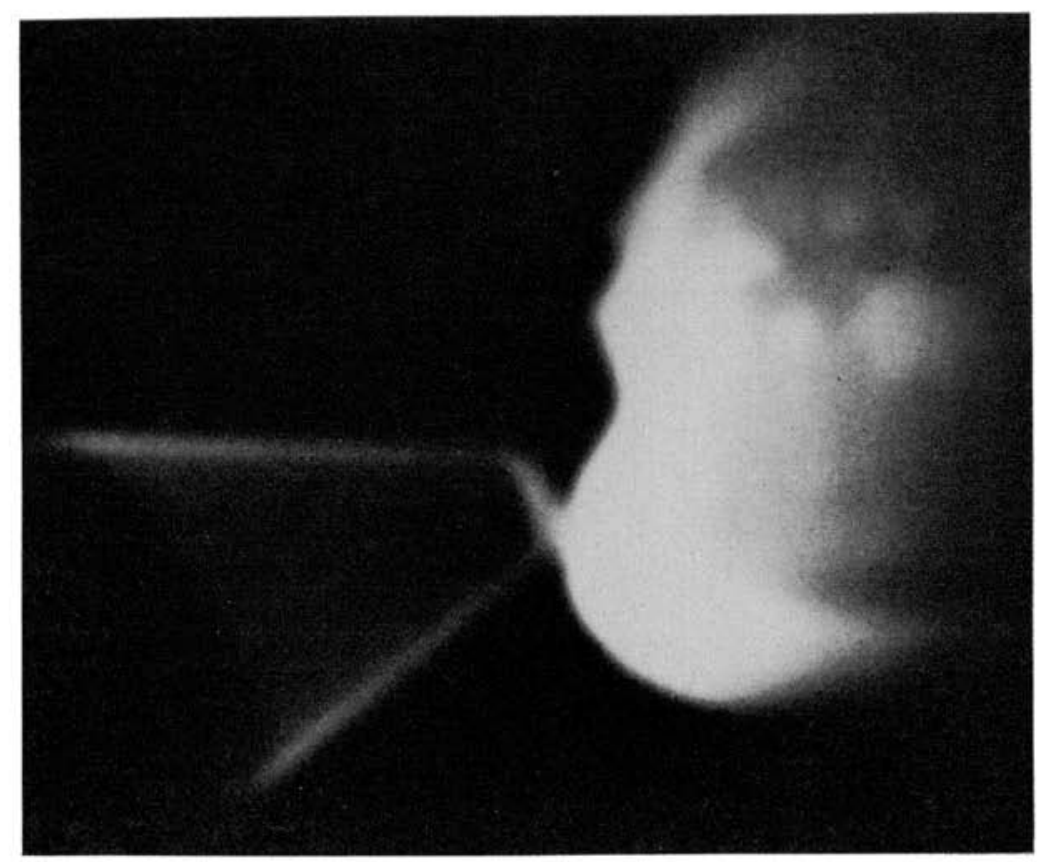

FIG. 10.-Radiograph of a phantom and dental X-ray apparatus produced with a pinhole camera. A periapical X-ray examination was made of the mandibular incisor region of the phantom, and this radiographic image was formed by the $\mathrm{X}$-radiation that scattered from the phantom and $\mathrm{X}$-ray apparatus to the pinhole camera located at the side of the phantom. The amount of scatter from the soft tissues of the phantom apparently is greater than that from the teeth and bones. 
of both anterior and posterior teeth are shown, thereby indicating that the scattered radiation, which reached the pinhole camera located in front of the phantom, came not only from superficial tissues but from deeper tissues as well.

\section{SUMMARY}

The design of a lead-walled pinhole camera, equipped with both photographic and radiographic films, was presented. The factors that controlled times of exposure and size and sharpness of the image were described mathematically. Photographs and radiographs that were produced simultaneously of dental subjects illustrated the usefulness of this new tool in radiation research in dentistry.

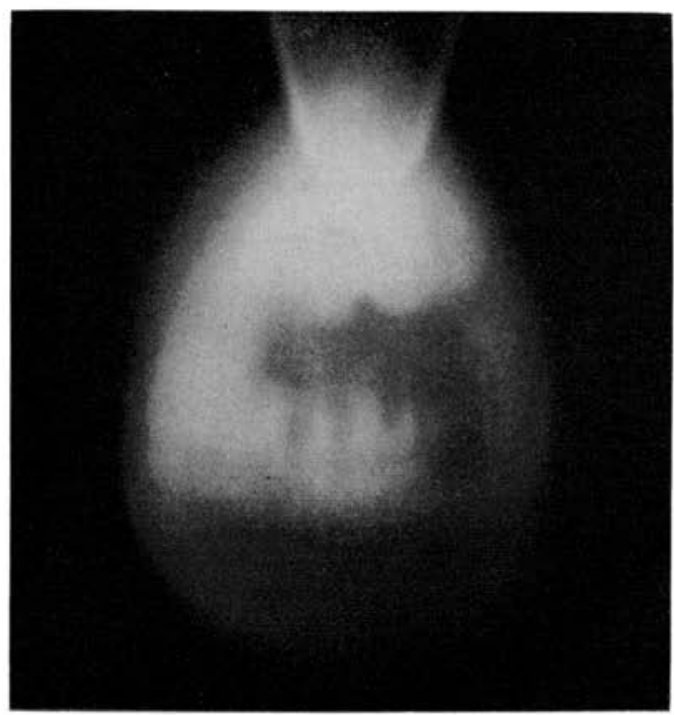

FIG. 11.-This is a radiograph made with scattered $\mathrm{X}$-radiation and a pinhole camera. It was made of a phantom on which a periapical X-ray examination was performed of the maxillary central incisor region. The camera was located in front of the phantom. The presence of the images of both anterior and posterior teeth indicates that the scattered radiation that produced the radiograph originated in both superficial and deep tissues.

\section{REFERENCES}

1. Anger, H. O. Use of Gamma-Ray Pinhole Camera for in Vivo Studies, Nature, 170:200, 1952.

2. . Scintillation Camera, Rev. Sci. Instr., 29:27, 1958.

3. Copeland, D. E., and Benjamin, E. W. Pinhole Camera for Gamma-Ray Sources, Nucleonics, $\mathbf{5}: 44,1949$.

4. Kemp, F. H., and Nichols, A. F. Focal Spot Sizes, Brit. J. Radiol., $31: 486,1958$.

5. KuntKe, A. H. G. On the Determination of Roentgen Tube Focal Spot Sizes by Pinhole Camera Roentgenography, Acta Radiol., 47:55, 1957.

6. Mortimer, R. K., Anger, H. O., and Tobias, C. A. The Gamma Ray Pinhole Camera with Image Amplifier, Inst. Rad. Eng., Convention Record, 2(pt. 9):2, 1954.

7. Odeblad, Erik. Pin-Hole Autoradiography, Acta Radiol., 43:145, 1955.

8. Payne, J. H. A Gamma Ray Pin-Hole Camera. Schenectady, N.Y.: Knolls Atomic Power Laboratory, June 13, 1958. (Processed.)

9. Preuss, L. E., and Jenkins, G. Pinhole Camera Maps Beta-active Deposits, Nucleonics, 16:98, 1958.

10. Richards, A. G. Radiation Protection via the Pinhole Camera, Oral Surg., Oral Med. \& Oral Path., $13: 953,1960$. 
11. Richards, A. G. A New Method for the Reduction of Gonadal Irradiation of Dental Patients, J.A.D.A. In press.

12. Roentgen, W. C. Ueber eine neue Art von Strahlen, Sitzungsb. d. Würzburg. Phys.-Med. Ges., p. 132, 1895. (Translated by Arthur Stanton, in Nature, $\mathbf{5 3 : 2 7 4 , 1 8 9 6 . )}$

13. Vuorinen, P. Reliability of Pinhole Methods in Evaluation of Radiation from Emission Area in Roentgen Tubes, Acta Radiol., 54:41, 1960.

14. Report of the International Commission on Radiological Units and Measurements. Method of Focal Spot Image and Measurement, Am. J. Roentgenol. Radium Therapy Nuclear Med., 85:191, 1961. 